Pacific Journal of Mathematics

THE METHOD OF INTERIOR PARALLELS APPLIED TO 


\title{
THE METHOD OF INTERIOR PARALLELS APPLIED TO POLYGONAL OR MULTIPLY CONNECTED MEMBRANES
}

\author{
JOSEPH HERSCH
}

\section{Introduction.}

1.1. The scope of this paper is (a) to discuss the possibilities of the method of interior parallels (Makai, Pólya, Payne-Weinberger) by considering the case of polygonal membranes $(\S 2) ;(b)$ to extend it to multiply connected domains in a more satisfactory manner than has hitherto been proposed (§3); to this end we use a result of $\mathrm{H}$. F. Weinberger [7] on the existence of an "effectless cut", published immediately after the present paper.

1.2. We consider the problem of a vibrating membrane covering a plane domain $G$ and fixed along the boundary $\Gamma$. We are interested in the first eigenvalue $\lambda_{1}$ of the problem $\Delta u+\lambda u=0$ in $G, u=0$ along $\Gamma$; by Rayleigh's principle,

$$
\lambda_{1} \leqq R[v] \equiv \frac{D(v)}{\iint_{\theta} v^{2} d A} \quad \text { if } v=0 \text { along } \Gamma .
$$

$d A=d x d y$ is the element of area; $D(v)=\iint_{\theta} g r a d^{2} v d A$, Dirichlet's integral; $R[v]$, Rayleigh's quotient.

The method of interior parallels consists in using trial functions $v$ whose level lines are parallel to $\Gamma$. It was first introduced by $\mathbf{E}$. Makai [2,3]: using the trial function $v(Q)=\delta_{Q \Gamma}(Q \in G, \delta=$ Euclidean distance), he obtained, for every simply or doubly connected membrane $G$ of area $A$, fixed along its boundary $\Gamma$ of total length $L_{r}$, the bound

$$
\lambda_{1} \leqq 3 \frac{L_{\Gamma}^{2}}{A^{2}} .
$$

His proof makes use of B. Sz.-Nagy's [6] inequality

$$
q(\delta) \leqq L_{\Gamma}
$$

bounding the total length $q(\delta)$ of the "interior parallel at distance $\delta$ " in a simply or doubly connected domain; as Sz.-Nagy proved, this length exists for almost all values of $\delta$. 
1.3. Refining Makai's method, G. Pólya [5] admits a priori for $v$ any regular function $v\left(\delta_{Q \Gamma}\right)$ satisfying $v(0)=0$.

Let us call $a=a(\delta)$ the area of the subdomain $\left\{Q \mid Q \in G, \delta_{Q \Gamma}<\delta\right\}$ of $G ; q(\delta)=d a / d \delta$. By Rayleigh's principle,

$$
\lambda_{1} \leqq R[v]=\frac{\int_{a=0}^{A}\left(\frac{d v}{d \delta}\right)^{2} d a}{\int_{a=0}^{A} v^{2} d a}=\frac{\int_{a=0}^{A} q^{2}\left(\frac{d v}{d a}\right)^{2} d a}{\int_{a=0}^{A} v^{2} d a} \text { if } v(0)=0 .
$$

Let $\lambda_{1}^{+}=\operatorname{Min}_{v(\delta)} R[v] ; \lambda_{1} \leqq \lambda_{1}^{+}$; if $G$ is simply or doubly connected inequality (2) gives

$$
\lambda_{1} \leqq \lambda_{1}^{+} \leqq \lambda_{\substack{1 \\ \text { Pólya }}}^{++} \equiv L_{\Gamma}^{2} \operatorname{Min}_{v(0)=0} \frac{\int_{a=0}^{A}\left(\frac{d v}{d a}\right)^{2} d a}{\int_{a=0}^{A} v^{2} d a}=\left(\frac{\pi}{2} \cdot \frac{L_{\Gamma}}{A}\right)^{2} ;
$$

this is Pólya's inequality (sharper than (1)).

1.4. For a simply connected domain $G$, L. E. Payne and H. F. Weinberger [4] made use of the sharp inequality of B. Sz.-Nagy [6]:

$$
q(\delta) \leqq L_{\Gamma}-2 \pi \delta
$$

it follows by integration that $q^{2} \leqq L_{\Gamma}^{2}-4 \pi a$ (see also [1]), whence by (3):

$$
\lambda_{1} \leqq \lambda_{1}^{+} \leqq \begin{gathered}
\lambda_{1}^{++} \\
P-W
\end{gathered}
$$

$$
\equiv \lambda_{\text {lext }}^{++}\left(A, L_{\Gamma}\right) \equiv \operatorname{Min}_{v(0)=0} \frac{\int_{a=0}^{A}\left(L_{\Gamma}^{2}-4 \pi a\right)\left(\frac{d v}{d a}\right)^{2} d a}{\int_{a=0}^{A} v^{2} d a}\left(\leqq \underset{\substack{\lambda_{1}^{\prime}(1) \mathrm{a} \\ \lambda^{++}}}{\mathrm{P}^{2}}\right) .
$$

Payne and Weinberger remarked that all inequalities (1), (2), (3), (4), (5), (6) remain valid if $G$ is allowed to have also interior boundary curves $\gamma$ along which the membrane is free ("holes"): $L_{\Gamma}$ is then the total length of the "fixed" boundaries $\Gamma, A$ the area of $G$ (without the holes); $q(\delta)$ is the length of that part of the "interior parallel" to $\Gamma$ (not $\gamma$ !) which lies inside $G$.

Inequality (4) is valid if $\Gamma$ is formed by the outer boundary $\Gamma_{0}$ and at most one inner boundary curve $\Gamma_{1}$; along the other interior boundary curves $\gamma_{2}, \gamma_{3}, \cdots, \gamma_{n}$ the membrane is free; $L_{\Gamma}=L_{\Gamma_{0}}+L_{\Gamma_{1}}$. -(5) and (6) are valid only if $\Gamma=\Gamma_{0}$ and all inner boundaries are free.

If $G$ is a circular ring fixed along its outer boundary $\Gamma_{0}$ and free along its inner boundary $\gamma_{1}$, its first eigenfunction $u_{1}=u_{1}(r)$, whence 
$\lambda_{1}=\lambda_{1}^{+}$, and $q^{2}=L_{\Gamma_{0}}^{2}-4 \pi a$, whence $\lambda_{1}^{+}=\underset{P-W}{\lambda_{1}^{++}}$. Therefore $\underset{P-W}{\lambda_{1}^{++}} \equiv$ $\lambda_{\text {lext }}^{++}\left(A, L_{\Gamma_{0}}\right)$ is equal to the first eigenvalue of an annular membrane fixed along $\Gamma_{0}$, free along $\gamma_{1}$.

$\lambda_{\text {lext }}^{++}\left(A, L_{r_{0}}\right)$ is the root of an equation involving Bessel functions; its solution is indicated graphically in Jahnke-Emde's Tables of functions, pp. 207-8.

The inequality $\lambda_{1} \leqq \lambda_{1-W}^{++}$thus expresses an "isoperimetric" extremal property of such annular membranes.

1.5. In another paper [1] one can find a "unified" and more detailed discussion of Makai's, Pólya's and Payne-Weinberger's methods; and furthermore the proof of an analogous "isoperimetric" theorem, which we shall essentially use in $\S 3$ :

Of all multiply connected membranes of given area $A$, fixed along one inner boundary Jordan curve $\Gamma_{1}$ of given length $L_{\Gamma_{1}}$ and free along all others ( $\gamma_{0}$ exterior; $\gamma_{2}, \gamma_{3}, \cdots, \gamma_{n}$ interior), the annulus has highest $\lambda_{1}$.

Let $\delta=\delta_{Q \Gamma_{1}}$ (Euclidean distance), and $q=q(\delta)$ as before; the proof of our theorem becomes easy once we introduce the new parameter

$$
t(\delta)=\int_{0}^{\delta} \frac{d \delta}{q}
$$

instead of $a(\delta)=\int_{0}^{\delta} q d \delta$ (see 1.3 and 1.4). We then have, instead of (3),

$$
\lambda_{1} \leqq R[v]=\frac{\int_{t=0}^{T}\left(\frac{d v}{d t}\right)^{2} d t}{\int_{t=0}^{T} q^{2} v^{2} d t} ; \quad \lambda_{1} \leqq \lambda_{1}^{+} \equiv \operatorname{Min}_{v} R[v] .
$$

(Often $T=\infty$.) This is the Rayleigh quotient of a vibrating string, fixed at its extremity $t=0$ and of total mass $\int_{t=0}^{T} q^{2} d t=A$.

B. Sz.-Nagy proved that here $q(\delta) \leqq L_{\Gamma_{1}}+2 \pi \delta$; whence by integration:

$$
q(t) \leqq L_{\Gamma_{1}} e^{2 \pi t} \quad \text { for } \quad t \leqq t_{1}=\frac{1}{4 \pi} \ln \left(1+\frac{4 \pi A}{L_{\Gamma_{1}}^{2}}\right) \quad \text { (see [1]) ; }
$$

the proof is completed by a discussion of the effect of displacing the masses along the vibrating string.-We thus have

$$
\lambda_{1} \leqq \lambda_{1}^{+} \leqq \lambda_{\text {lint }}^{++}\left(A, L_{\Gamma_{1}}\right),
$$

where $\lambda_{\text {1int }}^{++}\left(A, L_{\Gamma_{1}}\right)$ is the first eigenvalue of an annular membrane of 
area $A$, fixed along its interior boundary $\Gamma_{1}$ of length $L_{\Gamma_{1}}$, free along $\gamma_{0}$. To determine $\lambda_{\text {lint }}^{++}$, use again Jahnke-Emde's Tables of functions, pp. 207-8.

\section{Membranes with fixed polygonal outer boundary.}

2.1. For (simply or multiply connected) membranes, fixed along their polygonal outer boundary $\Gamma_{0}$ but free along the (possible) inner boundaries $\gamma_{1}, \gamma_{2}, \cdots, \gamma_{n}$, we shall sharpen Payne-Weinberger's upper bound (\$1.4).-Also, the new bounds obtained will give us a glimpse of the limits of the method's possibilities.

2.2. The regular polygon with $m$ sides, which is circumscribed to the unit circle, has perimeter $K_{m}=2 m t g(\pi / m)$, and area $K_{m} / 2$.

Any regular $m$-polygon with area $A$ and perimeter $L$, is circumscribed to a circle of radius $r_{i}=L / K_{m} ; L^{2}=\left(K_{m} r_{i}\right)^{2}=2 K_{m}\left(K_{m} r_{i}^{2} / 2\right)=$ $2 K_{m}\left(L r_{i} / 2\right)=2 K_{m} A$. Therefore, by the isoperimetric property of regular polygons, any $m$-polygon with area $A$ and perimeter $L$ satisfies

$$
L^{2} \geqq 2 K_{m} A \text {. }
$$

In particular, every $m$-polygon (whether convex or not), which is circumscribed to a circle of radius $r_{i}$, satisfies $A=L r_{i} / 2$; therefore

$$
L \geqq K_{m} r_{i} \text {. }
$$

Let $p \leqq m$; a regular $p$-polygon is an irregular $m$-polygon, circumscribed to the same circle, thus $K_{p} r_{i}=L \geqq K_{m} r_{i}$, whence $K_{p} \geqq K_{m} ; K_{m}$ is a decreasing function of $m$ (which can be verified directly); when $m \rightarrow \infty, K_{m} \searrow 2 \pi$.

2.3. Let the membrane cover a plane domain $G$ and be fixed only along the $m$-polygonal outer boundary $\Gamma_{0}$; let us call $\widetilde{G}(\supset G)$ the polygonal domain bounded by $\Gamma_{0}$; the line $\widetilde{\Gamma}_{0}^{(\delta)}$ parallel to $\Gamma_{0}$ in $\widetilde{G}$ is composed of $p \leqq m$ straight segments and possibly (if $\Gamma_{0}$ is not convex) some circular $\operatorname{arcs}$ of radius $\delta$. The length $\widetilde{q}(\delta)$ of $\widetilde{\Gamma}_{0}^{(\delta)}$ is a piecewise differentiable function of $\delta$; $\widetilde{q}(\delta) \geqq q(\delta)$. If the domain $\widetilde{G}^{(\delta)}$, bounded by $\widetilde{\Gamma}_{0}^{(\delta)}$, is convex, it is readily seen that, for $\varepsilon>0, \widetilde{q}(\delta)-\widetilde{q}(\delta+\varepsilon)$ is equal to the perimeter of a convex $p$-polygon (of sides parallel to $\Gamma_{0}$ ) with $p \leqq m$, circumscribed to a circle of radius $\varepsilon$; whence $\widetilde{q}(\delta)-\widetilde{q}(\delta+\varepsilon) \geqq$ $K_{p} \varepsilon \geqq K_{m} \varepsilon$. This remains true if $\widetilde{G}^{(\delta)}$ is non-convex: indeed, $\widetilde{q}(\delta)-\widetilde{q}(\delta+\varepsilon)$ is then larger than the perimeter of a non-convex $p$-polygon with $p \leqq m$, circumscribed to a circle of radius $\varepsilon$. We thus have always

$$
-\frac{d \widetilde{q}}{d \delta} \geqq K_{m} .
$$


As in 1.3 and 1.4, we use as parameter the area $a(\delta)=\int_{0}^{\delta} q d \delta$ of the subdomain $\left\{Q \mid Q \in G, \delta_{Q \Gamma_{0}}<\delta\right\} ; d a / d \delta=q$;

$$
\begin{aligned}
\frac{-d\left(\widetilde{q}^{2}\right)}{d a}=2 \widetilde{q}\left(\frac{-d \widetilde{q}}{d a}\right) \geqq 2 q\left(\frac{-d \widetilde{q}}{d a}\right) & =2 \frac{d a}{d \delta}\left(\frac{-d \widetilde{q}}{d a}\right) \\
& =2\left(\frac{-d \widetilde{q}}{d \delta}\right) \geqq 2 K_{m}
\end{aligned}
$$

whence by integration from 0 to $a: L_{T_{0}}^{2}-\widetilde{q}^{2} \geqq 2 K_{m} a$;

$$
q^{2} \leqq \widetilde{q}^{2} \leqq L_{\Gamma_{0}}^{2}-2 K_{m} a,
$$

with equality if $G=\widetilde{G}=$ regular $m$-polygon.-This evaluation (valid for $m$-polygons) is sharper than $q^{2} \leqq \widetilde{q}^{2} \leqq L_{\Gamma_{0}}^{2}-4 \pi a$ (always valid), which is the basis of Payne-Weinberger's method (see [1]).

Using (3), we thus may write (instead of (6)):

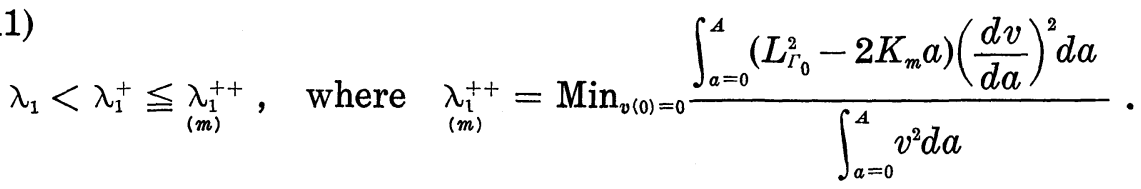

Note that for polygons $\lambda_{1}$ is always smaller than $\lambda_{1}^{+}$: this limits the sharpness obtainable by the method of interior parallels. When $m \rightarrow \infty, K_{m} \searrow 2 \pi$; thus

$$
\underset{(m)}{\lambda_{1}^{++}} \underset{(\infty)}{\lambda_{1}^{++}}=\underset{P-W}{\lambda_{1}^{++}}
$$

We shall construct an annular membrane having exactly the first eigenvalue $\underset{(m)}{\lambda_{1}^{++}}$:

Instead of $a$, we introduce a new independent variable $r$ by

$$
L_{T_{0}}^{2}-2 K_{m} a=K_{m}^{2} r^{2} \text {, i.e. } a=\frac{L_{T_{0}}^{2}}{2 K_{m}}-\frac{1}{2} K_{m} r^{2} ; \text { then } \frac{d a}{d r}=-K_{m} r \text {; }
$$

$$
\underset{\substack{\lambda_{1} \\(m)}}{++}=\operatorname{Min}_{v\left(R_{0}\right)=0} \frac{\int_{r=r_{1}}^{R_{0}}\left(\frac{d v}{d r}\right)^{2} K_{m} r d r}{\int_{r=r_{1}}^{R_{0}} v^{2} K_{m} r d r}=\operatorname{Min}_{v\left(R_{0}\right)=0} \frac{\int_{r=r_{1}}^{R_{U}}\left(\frac{d v}{d r}\right)^{2} 2 \pi r d r}{\int_{r=r_{1}}^{R_{0}} v^{2} 2 \pi r d r}
$$

with $R_{0}=L_{\Gamma_{0}} / K_{m}$ and $r_{1}^{2}=R_{0}^{2}-2 A / K_{m}$.

This is the annular membrane we wanted: fixed along its outer circle of radius $R_{0}$, free along its inner circle of radius $r_{1}$.-Consider two homothetic regular $m$-polygons, the outer one of length $L_{\Gamma_{0}}$, the inner one such that the area comprised between them be $A$ : the first is circumscribed to the circle of radius $R_{0}$, the second to the circle of radius $r_{1}$. 
REMARK. The fact that $\underset{(m)}{\lambda_{1}^{++}}$increases with $m$ thus expresses a property of Bessel functions.

2.4. More precise evaluations in terms of $A, L_{\Gamma_{0}}$ and the interior angles $\pi-\alpha_{1}, \pi-\alpha_{2}, \cdots, \pi-\alpha_{m}$ of $\Gamma_{0}$, when $\widetilde{G}$ is convex.

We consider a membrane $G$ fixed only along its convex polygonal outer boundary $\Gamma_{0}$. We have $\alpha_{1}+\cdots+\alpha_{m}=2 \pi, 0<\alpha_{i}<\pi$.

Let us call $F\left(\alpha_{1}, \cdots, \alpha_{m}\right)=2 \sum_{i=1}^{m} t g\left(\alpha_{i} / 2\right)$ the perimeter of the (convex) polygon $C$ with interior angles $\pi-\alpha_{1}, \cdots, \pi-\alpha_{m}$ (in this order), circumscribed to the unit circle. The area of $C$ is $F\left(\alpha_{1}, \cdots, \alpha_{m}\right) / 2$. By $\left(8^{\prime}\right), F\left(\alpha_{1}, \cdots, \alpha_{m}\right) \geqq K_{m}$; with equality if $\alpha_{1}=\cdots=\alpha_{m}=2 \pi / m$.

Every interior parallel $\widetilde{\Gamma}_{0}^{(\delta)}$ to $\Gamma_{0}$ in $\widetilde{G}$ is a polygon with $p \leqq m$ sides (parallel to those of $\Gamma_{0}$ ) and inner angles $\pi-\beta_{1}, \cdots, \pi-\beta_{p}$, where $\beta_{1}+\cdots+\beta_{p}=2 \pi$ and each $\beta_{j}$ is equal either to an $\alpha_{i}$ or to the sum of several consecutive $\alpha_{i}$. For a sufficiently small $\varepsilon>0$, $\widetilde{q}(\delta)-\widetilde{q}(\delta+\varepsilon)$ is equal to the length of a (convex) $p$-polygon with angles $\pi-\beta_{1}, \cdots, \pi-\beta_{p}$ (in this order), circumscribed to a circle of radius $\varepsilon$; whence $\widetilde{q}(\delta)-\widetilde{q}(\delta+\varepsilon)=F\left(\beta_{1}, \cdots, \beta_{p}\right) \cdot \varepsilon$;

$$
\frac{-d \widetilde{q}}{d \delta}=F\left(\beta_{1}, \cdots, \beta_{p}\right)=2 \sum_{j=1}^{p} t g \frac{\beta_{j}}{2}
$$

since $\widetilde{G}$ is by hypothesis convex, $0<\alpha_{i}<\pi, 0<\beta_{j}<\pi$, thus each $\operatorname{tg}\left(\alpha_{i} / 2\right)>0$ and

$$
\operatorname{tg} \frac{\alpha_{i}+\alpha_{i+1}}{2}=\frac{\operatorname{tg} \frac{\alpha_{i}}{2}+\operatorname{tg} \frac{\alpha_{i+1}}{2}}{1-\operatorname{tg} \frac{\alpha_{i}}{2} \operatorname{tg} \frac{\alpha_{i+1}}{2}}>\operatorname{tg} \frac{\alpha_{i}}{2}+\operatorname{tg} \frac{\alpha_{i+1}}{2}
$$

therefore $F\left(\beta_{1}, \cdots, \beta_{p}\right) \geqq F\left(\alpha_{1}, \cdots, \alpha_{m}\right)$ (which is also geometrically clear) and always

$$
-\frac{d \widetilde{q}}{d \delta} \geqq F\left(\alpha_{1}, \cdots, \alpha_{m}\right) ;
$$

whence

$$
q^{2} \leqq \widetilde{q}^{2} \leqq L_{\Gamma_{0}}^{2}-2 F\left(\alpha_{1}, \cdots, \alpha_{m}\right) a
$$

and the inequality 


$$
\lambda_{1}<\lambda_{1}^{+} \leqq \underset{\substack{\left(\alpha_{1}, \cdots, \alpha_{m}\right) \\ \lambda_{1}^{++}}}{\lambda^{\prime}} \text { where }
$$

$$
\underset{\left(\alpha_{1}, \cdots, \alpha_{m}\right)}{\lambda_{1}^{++}}=\operatorname{Min}_{v(0)=0} \frac{\int_{a=0}^{A}\left[L_{\Gamma_{0}}^{2}-2 F\left(\alpha_{1}, \cdots, \alpha_{m}\right) a\right]\left(\frac{d v}{d a}\right)^{2} d a}{\int_{a=0}^{4} v^{2} d a} .
$$

$\underset{\left(\alpha_{1}, \cdots, \alpha_{m}\right)}{\lambda_{1}^{+}} \leqq \underset{(m)}{\lambda_{1}^{++}}$; equality only if $\alpha_{1}=\cdots=\alpha_{m}=2 \pi / m$.

Let us now introduce another independent variable $r$ instead of a: $\quad L_{\Gamma_{0}}^{2}-2 F\left(\alpha_{1}, \cdots, \alpha_{m}\right) a=\left[F\left(\alpha_{1}, \cdots, \alpha_{m}\right)\right]^{2} \cdot r^{2}$; we then obtain a formula like (13) with $F\left(\alpha_{1}, \cdots, \alpha_{m}\right)$ instead of $K_{m}$, now $R_{0}=$ $L_{\Gamma_{0}} / F\left(\alpha_{1}, \cdots, \alpha_{m}\right)$ and $r_{1}^{2}=R_{0}^{2}-2 A / F\left(\alpha_{1}, \cdots, \alpha_{m}\right)$. The annular membrane with fixed outer circle of radius $R_{0}$ and free inner circle of radius $r_{1}$ has first eigenvalue $\underset{\left(\alpha_{1}, \cdots, \alpha_{m}\right)}{\lambda_{1}^{++}}$.

Let us construct two homothetic m-polygons, circumscribed to concentric circles, with sides parallel to those of $\Gamma_{0}$ (and in the same order), the outer polygon of length $L_{\Gamma_{0}}$, the inner polygon such that the area comprised between them be $A$; the outer circle has then radius $R_{0}$, the inner circle radius $r_{1}$ : this is our auxiliary annulus.

2.5. Remark on the limits of the possibilities of the method of interior parallels.-As follows from the above discussion, if $G=\widetilde{G}$ is itself a convex polygon circumscribed to a circle, we have $L_{\Gamma_{0}}^{2}=$ $2 F\left(\alpha_{1}, \cdots, \alpha_{m}\right) A$, whence $r_{1}=0 ; R_{0}=r_{\text {inser }}$;

$$
\begin{aligned}
\lambda_{1}<\underset{\left(\alpha_{1}, \cdots, \alpha_{m}\right)}{\lambda_{1}^{++}} & =\frac{j_{0}^{2}}{r_{\text {inser }}^{2}} \leqq \underset{(m)}{\lambda_{(m)}^{++}}<\underset{(\infty)}{\lambda_{1}^{++}}=\underset{P-W}{\lambda_{1}^{++}}<\underset{\text { Pólya }}{\lambda_{1}^{++}} \\
& =\left(\frac{\pi}{2} \frac{L_{\Gamma_{0}}}{A}\right)^{2}=\frac{\pi^{2}}{r_{\text {inser }}^{2}}<\underset{\text { Makai }}{\lambda_{1}^{++}}=3\left(\frac{L_{\Gamma_{0}}}{A}\right)^{2}=\frac{12}{r_{\text {inscr }}^{2}} .
\end{aligned}
$$

Observe that here $d \widetilde{q} / d \delta=-F\left(\alpha_{1}, \cdots, \alpha_{m}\right)$ and $q^{2}=\widetilde{q}^{2}=L_{T_{0}}^{2}-$ $2 F\left(\alpha_{1}, \cdots, \alpha_{m}\right) a$, i.e. $\lambda_{1}^{+}=\underset{\left(\alpha_{1}, \cdots, \alpha_{m}\right)}{\lambda_{1}^{++}}$is the first eigenvalue of the inscribed circle; the inequality $\lambda_{1}<j_{0}^{2} / r_{\text {inser }}^{2}$ is trivial (monotony), but the method of interior parallels is (in this case of a circumscribed polygon) unable to give any sharper bound.

It may be noted that Pólya's bound-and therefore Payne-Weinberger's bound as well as $\underset{(m)}{\lambda_{1}^{++}}$and $\underset{\left(\alpha_{1}, \cdots, \alpha_{m}\right)}{\lambda_{1}^{++}}$-become sharp for the infinite strip considered as the limit of a long rectangle: let $b$ be its breadth, $\underset{\text { Pólya }}{\lambda_{+}^{++}} \approx(\pi / b)^{2}$; but, if we consider the strip as the limit of a long rhombus (i.e. circumscribed to a circle), $\underset{\text { fólya }}{\lambda_{\text {folv }}^{++}} \approx(2 \pi / b)^{2}$ and $\underset{(\varepsilon, \pi-\varepsilon, \varepsilon, \pi-\varepsilon)}{\lambda_{1}^{++}}=j_{0}^{2} / r_{\text {inser }}^{2} \approx\left(2 j_{0} / b\right)^{2}$, which is trivial by monotony. 


\section{Multiply connected membranes.}

3.1. Let us consider e.g. a doubly connected membrane $G$, fixed both along its outer boundary $\Gamma_{0}$ and its inner boundary $\Gamma_{1}$.

(i) Given the area $A$ of $G$ and the lengths $L_{\Gamma_{0}}$ and $L_{\Gamma_{1}}$, we are looking for a bound $\lambda_{1} \leqq \lambda_{1}^{++}\left(A ; L_{\Gamma_{0}}, L_{\Gamma_{1}}\right)$ such that, when $\Gamma_{1}$ reduces to a point, $\lambda_{1}^{++}\left(A ; L_{\Gamma_{0}}, 0\right)=\lambda_{\text {lext }}^{++}\left(A, L_{\Gamma_{0}}\right)$ (exact bound of Payne-Weinberger); indeed, such is the case for the true $\lambda_{1}$.

This requirement is not fulfilled by Pólya's -or Makai's- bounds (even if $\Gamma_{1}$ is very small, they consider trial functions depending only on the distance to $\Gamma=\Gamma_{0} \cup \Gamma_{1}$, which does not correspond, qualitatively, to the behavior of the true first eigenfunction of $G$ ); nor is it fulfilled by Payne-Weinberger's suggestion to make $G$ simply connected by adding between $\Gamma_{0}$ and $\Gamma_{1}$ a rectilinear constraint (length $c)$, thus replacing $L_{\Gamma}$ by $L_{\Gamma}+2 c$ : indeed, when $\Gamma_{1}$ reduces to a point, this constraint would remain and the bound $\lambda_{\text {lext }}^{++}\left(A, L_{\Gamma_{0}}+L_{\Gamma_{1}}+2 c\right)$ would become $\lambda_{\text {lext }}^{++}\left(A, L_{\Gamma_{0}}+2 c\right)$ instead of $\lambda_{\text {lext }}^{++}\left(A, L_{\Gamma_{0}}\right)$. Any small boundary component $\Gamma_{1}$ has then a disproportionate effect on the bound.-In particular, consider a fixed annular membrane with radii 1 and $\varepsilon \rightarrow 0$; the true $\lambda_{1}$ tends to $j_{0}^{2} \cong 5.78 ; \lambda_{1}^{++}$(Payne-Weinberger) tends to $\lambda_{\text {lext }}^{++}(\pi, 2 \pi+2)$, which is larger than the first eigenvalue of the unit circular sector of aperture $360^{\circ}$, i.e. larger than $\pi^{2}$; Pólya's inequality gives (as for the circle) $\lambda_{1} \rightarrow \leqq((\pi / 2)(2 \pi / \pi))^{2}=\pi^{2}$.

(ii) We look for a bound which, for any fixed annular membrane, should coincide with the exact value $\lambda_{1}$.

3.2. From H. F. Weinberger's paper [7], which is printed immediately after the present one, it follows that: Given a multiply connected membrane $G$ which is fixed along its outer boundary $\Gamma_{0}$ and its inner boundary components $\Gamma_{1}, \Gamma_{2}, \cdots, \Gamma_{p}$, and free along its other inner boundaries $\gamma_{p+1}, \gamma_{p+2}, \cdots, \gamma_{n}$ (the $\Gamma_{i}$ are assumed to have continuous normals and the $\gamma_{j}$ to be analytic), then there exists an "effectless cutting" of the membrane $G$ into $p+1$ sub-membranes $G_{0}, G_{1}, \cdots, G_{p}$, where each $G_{i}$ has $\Gamma_{i}$ as a fixed boundary component and is otherwise free, such that $\lambda_{1}^{G_{0}}=\lambda_{1}^{\theta_{1}}=\cdots=\lambda_{1}^{\theta_{p}}=\lambda_{1}^{G}$. In other words: The domain $G$ can be cut into $G_{0}, \cdots, G_{p}$ by means of a system of analytic arcs along which $\partial u_{1} / \partial n=0$, where $u_{1}$ is the first eigenfunction of $G$; $u_{1}$ is then also the first eigenfunction of each $G_{i}$ (membrane fixed along $\Gamma_{i}$, free along the cuts and the $\gamma_{j}$ ). We use essentially this result in the following.

3.3. Let $A_{i}$ be the area of the partial domain $G_{i} ; A_{0}+A_{1}+$ $\cdots+A_{p}=A$; the lengths $L_{\Gamma_{0}}, L_{\Gamma_{1}}, \cdots, L_{\Gamma_{p}}$ are known, but not the individual $A_{i}$ ! -We know that $\lambda_{1} \leqq \lambda_{\text {lext }}^{++}\left(A_{0}, L_{\Gamma_{\mathrm{n}}}\right)$ and $\lambda_{1} \leqq \lambda_{\text {1int }}^{++}\left(A_{i}, L_{\Gamma_{i}}\right)$ 
for $i=1,2, \cdots, p$. Therefore:

$$
\lambda_{1} \leqq \min \left\{\lambda_{\text {lext }}^{++}\left(A_{0}, L_{\Gamma_{0}}\right) ; \lambda_{1 \mathrm{nt}}^{++}\left(A_{1}, L_{\Gamma_{1}}\right) ; \cdots ; \lambda_{\text {1int }}^{++}\left(A_{p}, L_{\Gamma_{p}}\right)\right\}
$$

and hence

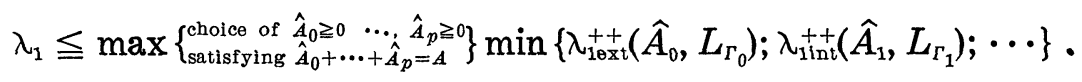

Since each of the $\lambda_{\text {lext }}^{++}, \lambda_{\text {lint }}^{++}$is a monotonous decreasing function of the corresponding $\hat{A}_{i}$, the max min is attained when $\hat{A}_{0}, \cdots, \hat{A}_{p}$ are chosen such that all those $\lambda_{1}^{++}$are equal:

$$
\lambda_{\text {lext }}^{++}\left(\hat{A}_{0}, L_{\Gamma_{0}}\right)=\lambda_{\text {1int }}^{++}\left(\hat{A}_{1}, L_{\Gamma_{1}}\right)=\cdots=\lambda_{\text {1int }}^{++}\left(\hat{A}_{p}, L_{\Gamma_{p}}\right) ;
$$

those are $p$ transcendental equations, which together with

$$
\hat{A_{0}}+\hat{A_{1}}+\cdots+\hat{A}_{p}=A
$$

determine $\hat{A}_{0}, \cdots, \hat{A_{p}}$; these values are in general NOT equal to the true $A_{0}, \cdots, A_{p}$ corresponding to Weinberger's "effectless cutting"; but the common value

$$
\lambda_{1}^{++}\left(\hat{A}_{i}, L_{\Gamma_{i}}\right)=\lambda_{1}^{++}\left(A ; L_{\Gamma_{0}}, L_{\Gamma_{1}}, \cdots, L_{\Gamma_{p}}\right)
$$

is the upper bound we were looking for.

Indeed: (i) If an inner boundary component $\Gamma_{p}$ reduces to a point, i.e. $L_{\Gamma_{p}} \rightarrow 0$, then the corresponding $\hat{A}_{p} \rightarrow 0$ (and also $A_{p} \rightarrow 0$ ); there remain $p-1$ transcendental relations in (14) between $\hat{A}_{0}, \cdots, \hat{A}_{p-1}$, which together with (15) determine these $p$ quantities; therefore $\lambda_{1}^{++}\left(A ; L_{\Gamma_{0}}, \cdots, L_{\Gamma_{p-1}}, 0\right)=\lambda_{1}^{++}\left(A ; L_{\Gamma_{0}}, \cdots, L_{\Gamma_{p-1}}\right)$ as we wanted.

In the special case $p=1$, we have $\lambda_{1}^{++}\left(A ; L_{\Gamma_{0}}, 0\right)=\lambda_{\text {lext }}^{++}\left(A, L_{\Gamma_{0}}\right)$.

(ii) If $p=1$ and $L_{\Gamma_{0}}^{2}-L_{\Gamma_{1}}^{2}=4 \pi A$, there exists a circular ring with area $A$, outer perimeter $L_{\Gamma_{0}}$ and inner perimeter $L_{\Gamma_{1}}$; its first eigenvalue is precisely equal to $\lambda_{1}^{++}\left(A ; L_{\Gamma_{0}}, L_{\Gamma_{1}}\right)$. (Here $\hat{A}_{0}=A_{0}$ and $\hat{A}_{1}=A_{1}, G_{0}$ and $G_{1}$ are separated by the "maximum line" of the annular membrane's first eigenfunction.)-Whence the isoperimetric inequality:

of all (doubly or multiply connected) membranes which are fixed along their outer boundary $\Gamma_{0}$ and one inner boundary component $\Gamma_{1}$ (and otherwise free), with given $A, L_{\Gamma_{0}}$ and $L_{\Gamma_{1}}$ satisfying $L_{\Gamma_{0}}^{2}-L_{\Gamma_{1}}^{2}$ $=4 \pi A$, the annular membrane has maximal $\lambda_{1}$.

ExAmple. A doubly connected fixed membrane, bounded by two circles of given radii, has maximum $\lambda_{1}$ when the circles are concentric. 
REMARKS. (a) If $\Gamma_{0}$ is a polygon, $\lambda_{\text {lext }}^{++}$in (14) can be advantageously replaced by $\underset{(m)}{\lambda_{1}^{++}}$or by $\underset{\left(\alpha_{1}, \cdots, \alpha_{m}\right)}{\lambda_{1}^{++}}$.

(b) If the considered membrane has a free outer boundary $\gamma_{0}$, the above discussion remains valid, the first term in (14) disappears from the formula, as disappear $A_{0}, \hat{A}_{0}$ and $L_{\Gamma_{0}}$.

My best thanks are due to $H$. F. Weinberger for his proof [7] of the existence of an "effectless cutting", which allowed the very simple proof given in this $\S 3$; without both Weinberger's kindness and skill, a long and delicate construction and discussion of a continuous trial function in the whole domain $G$ (with level lines consisting of arcs parallel to different $\Gamma_{i}$ ) would have been necessary to get the same (14), (15) and (16) finally.

\section{BIBLIOGRAPHY}

1. J. Hersch, Contribution to the method of interior parallels applied to vibrating membranes, Studies in Mathematical Analysis and Related Topics, Stanford University Press (1962), 132-139.

2. E. Makai, On the principal frequency of a convex membrane and related problems, Czechosl. Math. J., 9 (1959), 66-70.

3. - Bounds for the principal frequency of a membrane and the torsional rigidity of a beam, Acta Sci. Math. (=Acta Szeged), 20 (1959), 33-35.

4. L. E. Payne and H. F. Weinberger, Some isoperimetric inequalities for membrane frequencies and torsional rigidity, J. Math. Analysis and Appl., 2 (1961), 210-216.

5. G. Pólya, Two more inequalities between physical and geometrical quantities, J. Indian Math. Soc., 24 (1960), 413-419.

6. B. Sz.-Nagy, Ueber Parallelmengen nichtkonvexer ebener Bereiche, Acta Sci. Math. (=Acta Szeged), 20 (1959), 36-47.

7. H. F. Weinberger, An effectless cutting of a vibrating membrane, Pacific J. Math., 13 (1963), 1239-1240 (following immediately the present paper).

Battelle Memorial Institute, Geneva, Switzerland, AND SWiss Federal Institute of TeChNology, ZÜRICH. 


\section{PACIFIC JOURNAL OF MATHEMATICS}

\section{EDITORS}

RalPh S. Phillips

Stanford University

Stanford, California

M. G. Arsove

University of Washington

Seattle 5 , Washington
J. Dugundu

University of Southern California

Los Angeles 7, California

Lowell J. Paige

University of California

Los Angeles 24, California

\section{ASSOCIATE EDITORS}
E. F. BECKENBACH
D. DERRY
H. L. ROYDEN
E. G. STRAUS
T. M. CHERRY
M. OHTSUKA
E. SPANIER
F. WOLF

\section{SUPPORTING INSTITUTIONS}

\author{
UNIVERSITY OF BRITISH COLUMBIA \\ CALIFORNIA INSTITUTE OF TECHNOLOGY \\ UNIVERSITY OF CALIFORNIA \\ MONTANA STATE UNIVERSITY \\ UNIVERSITY OF NEVADA \\ NEW MEXICO STATE UNIVERSITY \\ OREGON STATE UNIVERSITY \\ UNIVERSITY OF OREGON \\ OSAKA UNIVERSITY \\ UNIVERSITY OF SOUTHERN CALIFORNIA
}

\author{
STANFORD UNIVERSITY \\ UNIVERSITY OF TOKYO \\ UNIVERSITY OF UTAH \\ WASHINGTON STATE UNIVERSITY \\ UNIVERSITY OF WASHINGTON \\ AMERICAN MATHEMATICAL SOCIETY \\ CALIFORNIA RESEARCH CORPORATION \\ SPACE TECHNOLOGY LABORATORIES \\ NAVAL ORDNANCE TEST STATION
}

Mathematical papers intended for publication in the Pacific Journal of Mathematrcs should be typewritten (double spaced), and the author should keep a complete copy. Manuscripts may be sent to any one of the four editors. All other communications to the editors should be addressed to the managing editor, L. J. Paige at the University of California, Los Angeles 24, California.

50 reprints per author of each article are furnished free of charge; additional copses may be obtained at cost in multiples of 50 .

The Pacific Journal of Mathematics is published quarterly, in March, June, September, and December. Effective with Volume 13 the price per volume (4 numbers) is $\$ 18.00$; single issues, $\$ 5.00$. Special price for current issues to individual faculty members of supporting institutions and to individual members of the American Mathematical Society: $\$ 8.00$ per volume; single issues $\$ 2.50$. Back numbers are available.

Subscriptions, orders for back numbers, and changes of address should be sent to Pacific Journal of Mathematics, 103 Highland Boulevard, Berkeley 8, California.

Printed at Kokusai Bunken Insatsusha (International Academic Printing Co., Ltd.), No. 6 , 2-chome, Fujimi-cho, Chiyoda-ku, Tokyo, Japan.

\section{PUBLISHED BY PACIFIC JOURNAL OF MATHEMATICS, A NON-PROFIT CORPORATION}

The Supporting Institutions listed above contribute to the cost of publication of this Journal, but they are not owners or publishers and have no responsibility for its content or policies. 


\section{Pacific Journal of Mathematics}

\section{Vol. 13, No. $4 \quad$ June, 1963}

Dallas O. Banks, Bounds for eigenvalues and generalized convexity ........... 1031

Jerrold William Bebernes, A subfunction approach to a boundary value problem for

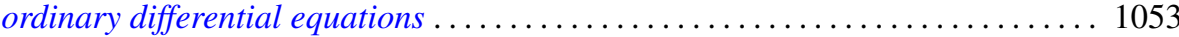

Woodrow Wilson Bledsoe and A. P. Morse, A topological measure construction . . . 1067

George Clements, Entropies of several sets of real valued functions . . . . . . . . . 1085

Sandra Barkdull Cleveland, Homomorphisms of non-commutative *-algebras . . . . . 1097

William John Andrew Culmer and William Ashton Harris, Convergent solutions of

ordinary linear homogeneous difference equations . . . . . . . . . . . . . . . 1111

Ralph DeMarr, Common fixed points for commuting contraction mappings . . . . . . 1139

James Robert Dorroh, Integral equations in normed abelian groups . . . . . . . . 1143

Adriano Mario Garsia, Entropy and singularity of infinite convolutions . . . . . . . 1159

J. J. Gergen, Francis G. Dressel and Wilbur Hallan Purcell, Jr., Convergence of extended Bernstein polynomials in the complex plane ................. 1171

Irving Leonard Glicksberg, A remark on analyticity of function algebras . . . . . . 1181

Charles John August Halberg, Jr., Semigroups of matrices defining linked operators

with different spectra ................................. 1187

Philip Hartman and Nelson Onuchic, On the asymptotic integration of ordinary

differential equations . . . . . . . . . . . . . . . . . . . . . . . . . . . . 1193

Isidore Heller, On a class of equivalent systems of linear inequalities . . . . . . . . . 1209

Joseph Hersch, The method of interior parallels applied to polygonal or multiply

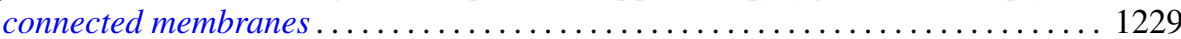

Hans F. Weinberger, An effectless cutting of a vibrating membrane . . . . . . . . . . 1239

Melvin F. Janowitz, Quantifiers and orthomodular lattices ....

Samuel Karlin and Albert Boris J. Novikoff, Generalized convex inequalities . .

Tilla Weinstein, Another conformal structure on immersed surfaces of negative

curvature.

Gregers Louis Krabbe, Spectral permanence of scalar operators

Shige Toshi Kuroda, Finite-dimensional perturbation and a representaion of

scattering operator.

Marvin David Marcus and Afton Herbert Cayford, Equality in certain

inequalities

Joseph Martin, A note on uncountably many disks .

Eugene Kay McLachlan, Extremal elements of the convex cone of semi-norms . . . . 1335

John W. Moon, An extension of Landau's theorem on tournaments . .

Louis Joel Mordell, On the integer solutions of $y(y+1)=x(x$

Kenneth Roy Mount, Some remarks on Fitting's invariants .....

Miroslav Novotný, Über Abbildungen von Mengen ............

Robert Dean Ryan, Conjugate functions in Orlicz spaces.

John Vincent Ryff, On the representation of doubly stochastic operators . . . . . . . . 1379

Donald Ray Sherbert, Banach algebras of Lipschitz functions .

James McLean Sloss, Reflection of biharmonic functions across analytic boundary

conditions with examples.

L. Bruce Treybig, Concerning homogeneity in totally ordered, connected topological space....

John Wermer, The space of real parts of a function algebra...

James Juei-Chin Yeh, Orthogonal developments of functionals and related theorems

in the Wiener space of functions of two variables......... 